Submitted to the Annals of Statistics

\title{
SUPPLEMENTARY MATERIAL FOR NOISY MATRIX DECOMPOSITION VIA CONVEX RELAXATION: OPTIMAL RATES IN HIGH DIMENSIONS
}

\author{
By Alekh Agarwal ${ }^{\star}$ AND Sahand N. Negahban ${ }^{\ddagger}$ AND \\ MARTIN J. WAINWRIGHT ${ }^{\dagger}$ \\ UC Berkeley, Department of EECS $S^{\star, \dagger}$ and Statistics ${ }^{\dagger}$ \\ MIT, Department of EECS $S^{\dagger}$
}

6. Simulation results. We have implemented the $M$-estimators based on the convex programs (10) and (14), in particular by adapting first-order optimization methods due to Nesterov [24]. In this section, we report simulation results that demonstrate the excellent agreement between our theoretical predictions and the behavior in practice. In all cases, we used square matrices $\left(d=d_{1}=d_{2}\right)$, and a stochastic noise matrix $W$ with i.i.d. $N\left(0, \frac{\nu^{2}}{d^{2}}\right)$ entries, with $\nu^{2}=1$. For any given rank $r$, we generated $\Theta^{\star}$ by randomly choosing the spaces of left and right singular vectors. We formed random sparse (elementwise or columnwise) matrices by choosing the positions of the non-zeros (entries or columns) uniformly at random.

Recall the estimator (10) from Example 4 for recovering a low-rank matrix $\Theta^{\star}$ corrupted by an arbitrary sparse matrix $\Gamma^{\star}$. In our first set of experiments, we fixed the matrix dimension $d=100$, and then studied a range of ranks $r$ for $\Theta^{\star}$, as well as a range of sparsity indices $s$ for $\Gamma^{\star}$. More specifically, we studied linear scalings of the form $r=\gamma d$ for a constant $\gamma \in(0,1)$, and $s=\beta d^{2}$ for a second constant $\beta \in(0,1)$.

Note that under this scaling, Corollary 2 predicts that the squared Frobenius error should be upper bounded as $c_{1} \gamma+c_{2} \beta \log (1 / \beta)$, for some universal constants $c_{1}, c_{2}$. Figure1(a) provides experimental confirmation of the accuracy of these theoretical predictions: varying $\gamma$ (with $\beta$ fixed) produces linear growth of the squared error as a function of $\gamma$. In Figure 1(b), we study the complementary scaling, with the rank ratio $\gamma$ fixed and the sparsity ratio $\beta$ varying in the interval $[.01, .1]$. Since $\beta \log (1 / \beta) \approx \Theta(\beta)$ over this interval, we should expect to see roughly linear scaling. Again, the plot shows good agreement with the theoretical predictions.

Now recall the estimator (14) from Example 5, designed for estimating a low-rank matrix plus a columnwise sparse matrix. We have observed similar linear dependence on the analogs of the parameters $\gamma$ and $\beta$, as predicted 


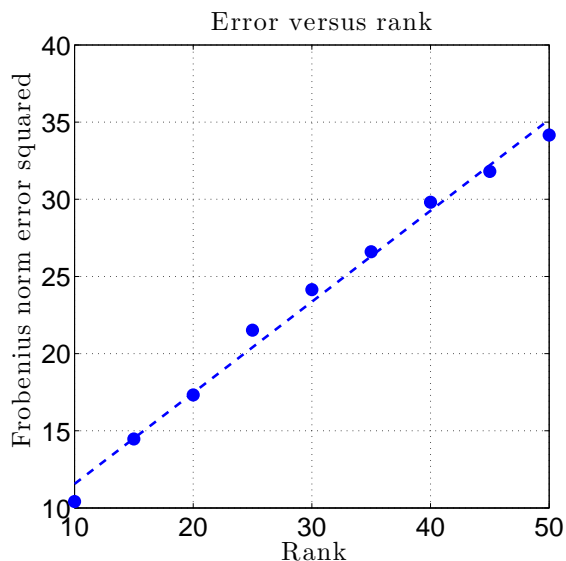

(a)

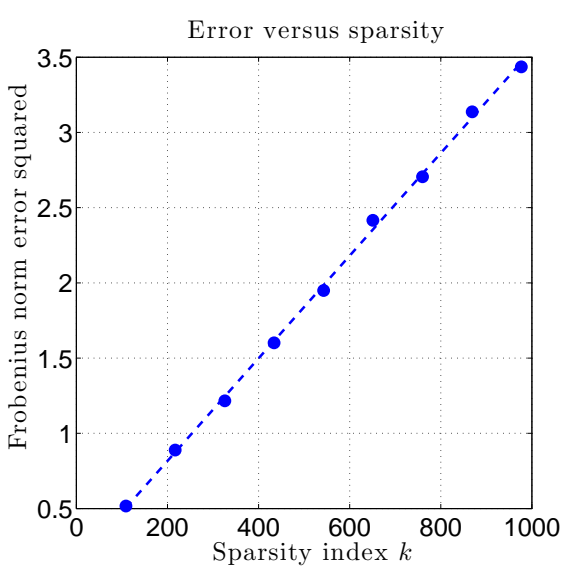

(b)

Fig 1. Behavior of the estimator (10). (a) Plot of the squared Frobenius error $e^{2}(\widehat{\Theta}, \widehat{\Gamma})$ versus the rank ratio $\gamma \in\{0.05: 0.05: 0.50\}$, for matrices of size $100 \times 100$ and $s=2171$ corrupted entries. The growth of the squared error is linear in $\gamma$, as predicted by the theory. (b) Plot of the squared Frobenius error $e^{2}(\widehat{\Theta}, \widehat{\Gamma})$ versus the sparsity parameter $\beta \in[0.01,0.1]$ for matrices of size $100 \times 100$ and rank $r=10$. Consistent with the theory, the squared error scales approximately linearly in $\beta$.

by our theory. In the interests of exhibiting a different phenomenon, here we report its performance for matrices of varying dimension, in all cases with $\Gamma^{\star}$ having $s=3 r$ non-zero columns. Figure 2(a) shows plots of squared Frobenius error versus the dimension for two choices of the rank $(r=10$ and $r=15)$, and the matrix dimension varying in the range $d \in\{100: 25: 300\}$. As predicted by our theory, these plots decrease at the rate $1 / d$. Indeed, this scaling is revealed by replotting the inverse squared error versus $d$, which produces the roughly linear plots shown in panel (b). Moreover, by comparing the relative slopes of these two curves, we see that the problem with rank $r=15$ requires roughly a dimension that is roughly $\frac{3}{2}$ larger than the problem with $r=10$ to achieve the same error. Again, this linear scaling in rank is consistent with Corollary 6 ,

7. Proofs. In this section, we provide the proofs of our main results, with the proofs of some more technical lemmas deferred to the appendices. 


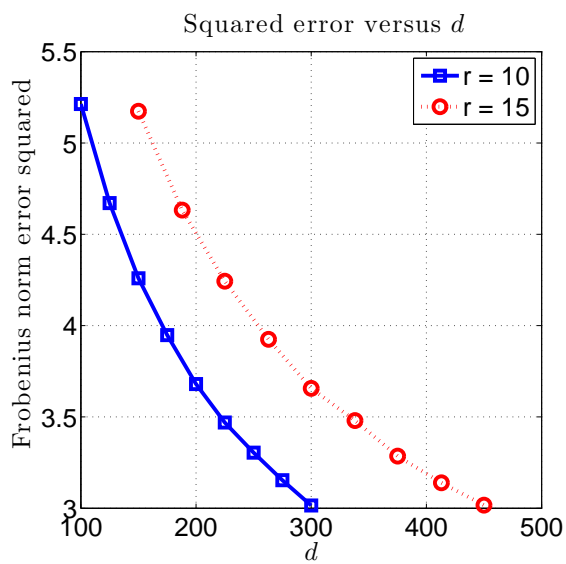

(a)

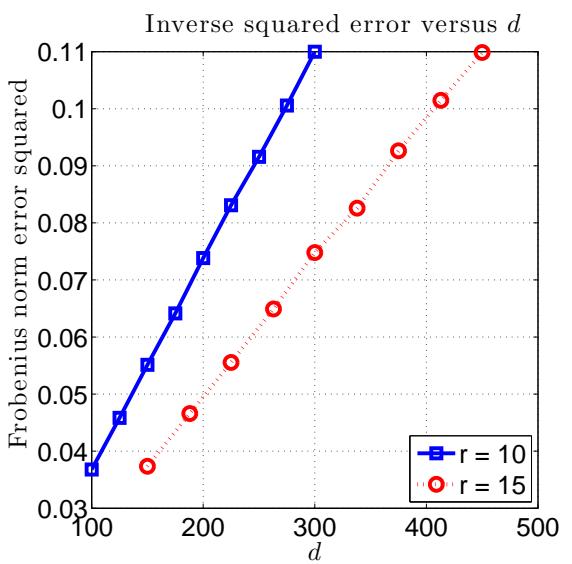

(b)

Fig 2. Behavior of the estimator (14). (a) Plot of the squared Frobenius error $e^{2}(\widehat{\Theta}, \widehat{\Gamma})$ versus the dimension $d \in\{100: 25: 300\}$, for two different choices of the rank $(r=10$ and $r=15)$. (b) Plot of the inverse squared Frobenius error versus the dimension, confirming the linear scaling in $d$ predicted by theory. Additionally, for $r=15$ we require a matrix dimension 1.5 times larger to reach the same error as $r=10$, consistent with theory.

7.1. Proof of Theorem 1. For the reader's convenience, let us recall here the two assumptions on the regularization parameters:

$$
\mu_{d} \geq 4 \mathcal{R}^{*}\left(\mathfrak{X}^{*}(W)\right)+\frac{4 \gamma \alpha}{\kappa_{d}}>0, \quad \text { and } \quad \lambda_{d} \geq 4\left\|\mathfrak{X}^{*}(W)\right\|_{\text {op }} .
$$

Furthermore, so as to simplify notation, let us define the error matrices $\widehat{\Delta}^{\Theta}:=\widehat{\Theta}-\Theta^{\star}$ and $\widehat{\Delta}^{\Gamma}:=\widehat{\Gamma}-\Gamma^{\star}$. Let $\left(\mathbb{M}, \mathbb{M}^{\perp}\right)$ denote an arbitrary subspace pair for which the regularizer $\mathcal{R}$ is decomposable. Throughout these proofs, we adopt the convenient shorthand notation $\widehat{\Delta}_{\mathbb{M}}^{\Gamma}:=\Pi_{\mathbb{M}}\left(\widehat{\Delta}^{\Gamma}\right)$ and $\widehat{\Delta}_{\mathbb{M}^{\perp}}^{\Gamma}=$ $\Pi_{\mathbb{M}^{\perp}}\left(\widehat{\Delta}^{\Gamma}\right)$, with similar definitions for $\Gamma^{\star} \mathbb{M}$ and $\Gamma^{\star} \mathbb{M}^{\perp}$.

We now turn to a lemma that deals with the behavior of the error matrices $\left(\widehat{\Delta}^{\Theta}, \widehat{\Delta}^{\Gamma}\right)$ when measured together using a weighted sum of the nuclear norm and regularizer $\mathcal{R}$. In order to state the following lemma, let us recall that for any positive $\left(\mu_{d}, \lambda_{d}\right)$, the weighted norm is defined as $\mathcal{Q}(\Theta, \Gamma):=\|\Theta\|_{\mathrm{N}}+\frac{\mu_{d}}{\lambda_{d}} \mathcal{R}(\Gamma)$.

With this notation, we have the following:

Lemma 1. For any $r=1,2, \ldots, d$, there is a decomposition $\widehat{\Delta}^{\Theta}=\widehat{\Delta}_{A}^{\Theta}+$ $\widehat{\Delta}_{B}^{\Theta}$ such that: 
(a) The decomposition satisfies

$$
\operatorname{rank}\left(\widehat{\Delta}_{A}^{\Theta}\right) \leq 2 r, \quad \text { and } \quad\left(\widehat{\Delta}_{A}^{\Theta}\right)^{T} \widehat{\Delta}_{B}^{\Theta}=\left(\widehat{\Delta}_{B}^{\Theta}\right)^{T} \widehat{\Delta}_{A}^{\Theta}=0
$$

(b) The difference $\mathcal{Q}\left(\Theta^{\star}, \Gamma^{\star}\right)-\mathcal{Q}\left(\Theta^{\star}+\widehat{\Delta}^{\Theta}, \Gamma^{\star}+\widehat{\Delta}^{\Gamma}\right)$ is upper bounded by

$$
\mathcal{Q}\left(\widehat{\Delta}_{A}^{\Theta}, \widehat{\Delta}_{\mathbb{M}}^{\Gamma}\right)-\mathcal{Q}\left(\widehat{\Delta}_{B}^{\Theta}, \widehat{\Delta}_{\mathbb{M} \perp}^{\Gamma}\right)+2 \sum_{j=r+1}^{d} \sigma_{j}\left(\Theta^{\star}\right)+\frac{2 \mu_{d}}{\lambda_{d}} \mathcal{R}\left(\Gamma^{\star} \mathbb{M}^{\perp}\right) .
$$

(c) Under conditions (52) on $\mu_{d}$ and $\lambda_{d}$, the error matrices $\widehat{\Delta}^{\Theta}$ and $\widehat{\Delta}^{\Gamma}$ satisfy

$$
\mathcal{Q}\left(\widehat{\Delta}_{B}^{\Theta}, \widehat{\Delta}_{\mathbb{M}^{\perp}}^{\Gamma}\right) \leq 3 \mathcal{Q}\left(\widehat{\Delta}_{A}^{\Theta}, \widehat{\Delta}_{\mathbb{M}}^{\Gamma}\right)+4\left\{\sum_{j=r+1}^{d} \sigma_{j}\left(\Theta^{\star}\right)+\frac{\mu_{d}}{\lambda_{d}} \mathcal{R}\left(\Gamma^{\star}{ }_{\mathbb{M}^{\perp}}\right)\right\} .
$$

for any $\mathcal{R}$-decomposable pair $\left(\mathbb{M}, \mathbb{M}^{\perp}\right)$.

See Appendix 8 for the proof of this result.

Our second lemma guarantees that the cost function $\mathcal{L}(\Theta, \Gamma)=\frac{1}{2} \| Y-$ $\mathfrak{X}(\Theta+\Gamma) \|_{F}^{2}$ is strongly convex in a restricted set of directions. In particular, if we let $\delta \mathcal{L}\left(\widehat{\Delta}^{\Theta}, \widehat{\Delta}^{\Gamma}\right)$ denote the error in the first-order Taylor series expansion around $\left(\Theta^{\star}, \Gamma^{\star}\right)$, then some algebra shows that

$$
\delta \mathcal{L}\left(\widehat{\Delta}^{\Theta}, \widehat{\Delta}^{\Gamma}\right)=\frac{1}{2}\left\|\mathfrak{X}\left(\widehat{\Delta}^{\Theta}+\widehat{\Delta}^{\Gamma}\right)\right\|_{\mathrm{F}}^{2} .
$$

The following lemma shows that (up to a slack term) this Taylor error is lower bounded by the squared Frobenius norm.

Lemma 2 (Restricted strong convexity). Under the conditions of Theorem 1, the first-order Taylor series error (55) is lower bounded by

$$
\frac{\gamma}{4}\left(\left\|\widehat{\Delta}^{\Theta}\right\|_{\mathrm{F}}^{2}+\left\|\widehat{\Delta}^{\Gamma}\right\|_{\mathrm{F}}^{2}\right)-\frac{\lambda_{d}}{2} \mathcal{Q}\left(\widehat{\Delta}^{\Theta}, \widehat{\Delta}^{\Gamma}\right)-16 \tau_{n}\left\{\sum_{j=r+1}^{d} \sigma_{j}\left(\Theta^{\star}\right)+\frac{\mu_{d}}{\lambda_{d}} \mathcal{R}\left(\Gamma^{\star}{ }_{\mathbb{M}^{\perp}}\right)\right\}^{2} .
$$

We prove this result in Appendix 9

Using Lemmas 1 and 2, we can now complete the proof of Theorem 11 By the optimality of $(\widehat{\Theta}, \widehat{\Gamma})$ and the feasibility of $\left(\Theta^{\star}, \Gamma^{\star}\right)$, we have

$$
\begin{gathered}
\frac{1}{2}\|Y-\mathfrak{X}(\widehat{\Theta}+\widehat{\Gamma})\|_{\mathrm{F}}^{2}+\lambda_{d}\|\widehat{\Theta}\|_{\mathrm{N}}+\mu_{d} \mathcal{R}(\widehat{\Gamma}) \leq \frac{1}{2}\left\|Y-\mathfrak{X}\left(\Theta^{\star}+\Gamma^{\star}\right)\right\|_{\mathrm{F}}^{2}+\lambda_{d}\left\|\Theta^{\star}\right\|_{\mathrm{N}}+\mu_{d} \mathcal{R}\left(\Gamma^{\star}\right) . \\
\text { imsart-aos ver. 2007/12/10 file: A0S_supp.tex date: June 5, } 2012
\end{gathered}
$$


Recalling that $Y=\mathfrak{X}\left(\Theta^{\star}+\Gamma^{\star}\right)+W$, and re-arranging in terms of the errors $\widehat{\Delta}^{\Theta}=\widehat{\Theta}-\Theta^{\star}$ and $\widehat{\Delta}^{\Gamma}=\widehat{\Gamma}-\Gamma^{\star}$, we obtain $\frac{1}{2}\left\|\mathfrak{X}\left(\widehat{\Delta}^{\Theta}+\widehat{\Delta}^{\Gamma}\right)\right\|_{\mathrm{F}}^{2} \leq\left\langle\left\langle\widehat{\Delta}^{\Theta}+\widehat{\Delta}^{\Gamma}, \mathfrak{X}^{*}(W)\right\rangle\right\rangle+\lambda_{d} \mathcal{Q}\left(\Theta^{\star}, \Gamma^{\star}\right)-\lambda_{d} \mathcal{Q}\left(\Theta^{\star}+\widehat{\Delta}^{\Theta}, \Gamma^{\star}+\widehat{\Delta}^{\Gamma}\right)$,

where the weighted norm $\mathcal{Q}$ was previously defined (20).

We now substitute inequality (53) from Lemma 1 into the right-hand-side of the above equation to obtain

$$
\begin{array}{r}
\frac{1}{2}\left\|\mathfrak{X}\left(\widehat{\Delta}^{\Theta}+\widehat{\Delta}^{\Gamma}\right)\right\|_{\mathrm{F}}^{2} \leq\left\langle\left\langle\widehat{\Delta}^{\Theta}+\widehat{\Delta}^{\Gamma}, \mathfrak{X}^{*}(W)\right\rangle+\lambda_{d} \mathcal{Q}\left(\widehat{\Delta}_{A}^{\Theta}, \widehat{\Delta}_{\mathbb{M}}^{\Gamma}\right)-\lambda_{d} \mathcal{Q}\left(\widehat{\Delta}_{B}^{\Theta}, \widehat{\Delta}_{\mathbb{M} \perp}^{\Gamma}\right)\right. \\
+2 \lambda_{d} \sum_{j=r+1}^{d} \sigma_{j}\left(\Theta^{\star}\right)+2 \mu_{d} \mathcal{R}\left(\Gamma^{\star}{ }_{\mathbb{M}^{\perp}}\right)
\end{array}
$$

Some algebra and an application of Hölder's inequality and the triangle inequality allows us to obtain the upper bound

$$
\begin{aligned}
& \left(\left\|\widehat{\Delta}_{A}^{\Theta}\right\|_{\mathrm{N}}+\left\|\widehat{\Delta}_{B}^{\Theta}\right\|_{\mathrm{N}}\right)\left\|\mathfrak{X}^{*}(W)\right\|_{\mathrm{op}}+\left(\mathcal{R}\left(\widehat{\Delta}_{\mathbb{M}^{\Gamma}}^{\Gamma}\right)+\mathcal{R}\left(\widehat{\Delta}_{\mathbb{M}^{\perp}}^{\Gamma}\right)\right) \mathcal{R}^{*}\left(\mathfrak{X}^{*}(W)\right) \\
& -\lambda_{d} \mathcal{Q}\left(\widehat{\Delta}_{B}^{\Theta}, \widehat{\Delta}_{\mathbb{M}^{\perp}}^{\Gamma}\right)+\lambda_{d} \mathcal{Q}\left(\widehat{\Delta}_{A}^{\Theta}, \widehat{\Delta}_{\mathbb{M}^{M}}^{\Gamma}\right)+2 \lambda_{d} \sum_{j=r+1}^{d} \sigma_{j}\left(\Theta^{\star}\right)+2 \mu_{d} \mathcal{R}\left(\Gamma^{\star} \mathbb{M}^{\perp}\right) .
\end{aligned}
$$

Recalling conditions (52) for $\mu_{d}$ and $\lambda_{d}$, we obtain the inequality

$$
\frac{1}{2}\left\|\mathfrak{X}\left(\widehat{\Delta}^{\Theta}+\widehat{\Delta}^{\Gamma}\right)\right\|_{\mathrm{F}}^{2} \leq \frac{3 \lambda_{d}}{2} \mathcal{Q}\left(\widehat{\Delta}_{A}^{\Theta}, \widehat{\Delta}_{\mathbb{M}}^{\Gamma}\right)+2 \lambda_{d} \sum_{j=r+1}^{d} \sigma_{j}\left(\Theta^{\star}\right)+2 \mu_{d} \mathcal{R}\left(\Gamma^{\star}{ }_{\mathbb{M}}^{\perp}\right) .
$$

Using inequality (56) from Lemma 2 to lower bound the right-hand side, and then rearranging terms yields

$$
\begin{aligned}
& \text { (57) } \frac{\gamma}{4}\left(\left\|\widehat{\Delta}^{\Theta}\right\|_{\mathrm{F}}^{2}+\left\|\widehat{\Delta}^{\Gamma}\right\|_{\mathrm{F}}^{2}\right) \leq \frac{3 \lambda_{d}}{2} \mathcal{Q}\left(\widehat{\Delta}_{A}^{\Theta}, \widehat{\Delta}_{\mathbb{M}}^{\Gamma}\right)+\frac{\lambda_{d}}{2} \mathcal{Q}\left(\widehat{\Delta}^{\Theta}, \widehat{\Delta}^{\Gamma}\right) \\
& +16 \tau_{n}\left\{\sum_{j=r+1}^{d} \sigma_{j}\left(\Theta^{\star}\right)+\frac{\mu_{d}}{\lambda_{d}} \mathcal{R}\left(\Gamma^{\star} \mathbb{M}^{\perp}\right)\right\}^{2}+2 \lambda_{d} \sum_{j=r+1}^{d} \sigma_{j}\left(\Theta^{\star}\right)+2 \mu_{d} \mathcal{R}\left(\Gamma^{\star}{ }_{\mathbb{M}^{\perp}}\right) .
\end{aligned}
$$

Now note that by the triangle inequality $\mathcal{Q}\left(\widehat{\Delta}^{\Theta}, \widehat{\Delta}^{\Gamma}\right) \leq \mathcal{Q}\left(\widehat{\Delta}_{A}^{\Theta}, \widehat{\Delta}_{\mathbb{M}}^{\Gamma}\right)+\mathcal{Q}\left(\widehat{\Delta}_{B}^{\Theta}, \widehat{\Delta}_{\mathbb{M} \perp}^{\Gamma}\right)$, so that combined with the bound (53) from Lemma 1, we obtain

$$
\mathcal{Q}\left(\widehat{\Delta}^{\Theta}, \widehat{\Delta}^{\Gamma}\right) \leq 4 \mathcal{Q}\left(\widehat{\Delta}_{A}^{\Theta}, \widehat{\Delta}_{\mathbb{M}}^{\Gamma}\right)+4\left\{\sum_{j=r+1}^{d} \sigma_{j}\left(\Theta^{\star}\right)+\frac{\mu_{d}}{\lambda_{d}} \mathcal{R}\left(\Gamma^{\star} \mathbb{M}^{\perp}\right)\right\}
$$


Substituting this upper bound into equation (57) yields

$$
\begin{aligned}
& \text { (58) } \frac{\gamma}{4}\left(\left\|\widehat{\Delta}^{\Theta}\right\|_{\mathrm{F}}^{2}+\left\|\widehat{\Delta}^{\Gamma}\right\|_{\mathrm{F}}^{2}\right) \leq 4 \mathcal{Q}\left(\widehat{\Delta}_{A}^{\Theta}, \widehat{\Delta}_{\mathbb{M}}^{\Gamma}\right) \\
& +16 \tau_{n}\left\{\sum_{j=r+1}^{d} \sigma_{j}\left(\Theta^{\star}\right)+\frac{\mu_{d}}{\lambda_{d}} \mathcal{R}\left(\Gamma^{\star}{ }_{\mathbb{M}^{\perp}}\right)\right\}^{2}+4\left\{\lambda_{d} \sum_{j=r+1}^{d} \sigma_{j}\left(\Theta^{\star}\right)+\mu_{d} \mathcal{R}\left(\Gamma^{\star}{ }_{\mathbb{M}^{\perp}}\right)\right\} .
\end{aligned}
$$

Noting that $\widehat{\Delta}_{A}^{\Theta}$ has rank at most $2 r$ and that $\widehat{\Delta}_{\mathbb{M}}^{\Gamma}$ lies in the model space $\mathbb{M}$, we find that

$$
\begin{aligned}
\lambda_{d} \mathcal{Q}\left(\widehat{\Delta}_{A}^{\Theta}, \widehat{\Delta}_{\mathbb{M}}^{\Gamma}\right) & \leq \sqrt{2 r} \lambda_{d}\left\|\widehat{\Delta}_{A}^{\Theta}\right\|_{\mathrm{F}}+\Psi(\mathbb{M}) \mu_{d}\left\|\widehat{\Delta}_{\mathbb{M}}^{\Gamma}\right\|_{\mathrm{F}} \\
& \leq \sqrt{2 r} \lambda_{d}\left\|\widehat{\Delta}^{\Theta}\right\|_{\mathrm{F}}+\Psi(\mathbb{M}) \mu_{d}\left\|\widehat{\Delta}^{\Gamma}\right\|_{\mathrm{F}} .
\end{aligned}
$$

Substituting the above inequality into equation (58) and rearranging the terms involving $e^{2}\left(\widehat{\Delta}^{\Theta}, \widehat{\Delta}^{\Gamma}\right)$ yields the claim.

We next present the proofs for Corollaries 2 and 4 . In order to do a combined treatment for both, we considered a rescaled model in Corollary 4 where we map $X \mapsto X / \sqrt{n}$ and $W \mapsto W / \sqrt{n}$. With this transformation, we observe that $W \sim N\left(0, \nu^{2} / n\right)$ and $\sigma_{\min }, \sigma_{\max }$ are the eigenvalues of this rescaled matrix $X$.

7.2. Proof of Corollaries 囷 and 4 Note that with the above rescalings, Corollary 2 can be viewed as a special case of Corollary 4 , in which $n=d_{1}$ and $X=I_{d_{1} \times d_{1}}$. Consequently, we may prove the latter result, and then obtain the former result with this specialization. Recall that we let $\sigma_{\min }$ and $\sigma_{\max }$ denote (respectively) the minimum and maximum eigenvalues of $X$, and that $\kappa_{\max }=\max _{j=1, \ldots, d_{1}}\left\|X_{j}\right\|_{2}$ denotes the maximum $\ell_{2}$-norm over the columns. (In the special case $X=I_{d_{1} \times d_{2}}$, we have $\sigma_{\min }=\sigma_{\max }=\kappa_{\max }=1$.)

Both corollaries are based on the regularizer, $\mathcal{R}(\cdot)=\|\cdot\|_{1}$, and the associated dual norm $\mathcal{R}^{*}(\cdot)=\|\cdot\|_{\infty}$. We need to verify that the stated choices of $\left(\lambda_{d}, \mu_{d}\right)$ satisfy the requirements (29) of Corollary 1, Given our assumptions on the pair $(X, W)$, a little calculation shows that the matrix $Z:=X^{T} W \in \mathbb{R}^{d_{1} \times d_{2}}$ has independent columns, with each column $Z_{j} \sim N\left(0, \nu^{2} \frac{X^{T} X}{n}\right)$. Since $\left\|X^{T} X\right\|_{\text {op }} \leq \sigma_{\max }^{2}$, known results on the singular values of Gaussian random matrices [1] imply that

$$
\mathbb{P}\left[\left\|X^{T} W\right\|_{\text {op }} \geq \frac{4 \nu \sigma_{\max }\left(\sqrt{d_{1}}+\sqrt{d_{2}}\right)}{\sqrt{n}}\right] \leq 2 \exp \left(-c\left(d_{1}+d_{2}\right)\right) .
$$

Consequently, setting $\lambda_{d} \geq \frac{16 \nu \sigma_{\max }\left(\sqrt{d_{1}}+\sqrt{d_{2}}\right)}{\sqrt{n}}$ ensures that the requirement (26) is satisfied. As for the associated requirement for $\mu_{d}$, it suffices to upper 
bound the elementwise $\ell_{\infty}$ norm of $X^{T} W$. Since the $\ell_{2}$ norm of the columns of $X$ are bounded by $\kappa_{\max }$, the entries of $X^{T} W$ are i.i.d. and Gaussian with variance at most $\left(\nu \kappa_{\max }\right)^{2} / n$. Consequently, the standard Gaussian tail bound combined with union bound yields

$$
\mathbb{P}\left[\left\|X^{T} W\right\|_{\infty} \geq 4 \frac{\nu \kappa_{\max }}{\sqrt{n}} \log \left(d_{1} d_{2}\right)\right] \leq \exp \left(-\log d_{1} d_{2}\right),
$$

from which we conclude that the stated choices of $\left(\lambda_{d}, \mu_{d}\right)$ are valid with high probability. Turning now to the RSC condition, we note that in the case of multivariate regression, we have

$$
\frac{1}{2}\|\mathfrak{X}(\Delta)\|_{\mathrm{F}}^{2}=\frac{1}{2}\|X \Delta\|_{\mathrm{F}}^{2} \geq \frac{\sigma_{\min }^{2}}{2}\|\Delta\|_{\mathrm{F}}^{2},
$$

showing that the RSC condition holds with $\gamma=\sigma_{\text {min }}^{2}$.

In order to obtain the sharper result for $X=I_{d_{1} \times d_{1}}$ in Corollary 2 -in which $\log \left(d_{1} d_{2}\right)$ is replaced by the smaller quantity $\log \left(\frac{d_{1} d_{2}}{s}\right)$ - we need to be more careful in upper bounding the noise term $\left\langle\left\langle W, \widehat{\Delta}^{\Gamma}\right\rangle\right\rangle$. We refer the reader to Appendix 10.1 for details of this argument.

7.3. Proof of Corollary 3. For this model, the noise matrix is recentered Wishart noise - namely, $W=\frac{1}{n} \sum_{i=1}^{n} Z_{i} Z_{i}^{T}-\Sigma$, where each $Z_{i} \sim N(0, \Sigma)$. Letting $U_{i} \sim N\left(0, I_{d \times d}\right)$ be i.i.d. Gaussian random vectors, we have

$\|W\|_{\mathrm{op}}=\left\|\sqrt{\Sigma}\left(\frac{1}{n} \sum_{i=1}^{n} U_{i} U_{i}-I_{d \times d}\right) \sqrt{\Sigma}\right\|_{\mathrm{op}} \leq\|\Sigma\|_{\mathrm{op}}\left\|\frac{1}{n} \sum_{i=1}^{n} U_{i} U_{i}^{T}-I_{d \times d}\right\|_{\mathrm{op}} \leq 4\|\Sigma\|_{\mathrm{op}} \sqrt{\frac{d}{n}}$,

where the final bound holds with probability greater than $1-2 \exp \left(-c_{1} d\right)$, using standard tail bounds on Gaussian random matrices [11]. Thus, we see that the specified choice (36) of $\lambda_{d}$ is valid for Theorem 1 with high probability.

We now turn to the choice of $\mu_{d}$. The entries of $W$ are products of Gaussian variables, and hence have sub-exponential tails (e.g., [4]). Therefore, for any entry $(i, j)$, we have the tail bound $\mathbb{P}\left[\left|W_{i j}\right|>\rho(\Sigma) t\right] \leq 2 \exp \left(-n t^{2} / 20\right)$, valid for all $t \in(0,1]$. By union bound over all $d^{2}$ entries, we conclude that

$$
\mathbb{P}\left[\|W\|_{\infty} \geq 8 \rho(\Sigma) \sqrt{\frac{\log d}{n}}\right] \leq 2 \exp \left(-c_{2} \log d\right),
$$

which shows that the specified choice of $\mu_{d}$ is also valid with high probability. 
7.4. Proof of Proposition 1. To begin, let us recall condition (52) on the regularization parameters, and that, for this proof, the matrices $(\widehat{\Theta}, \widehat{\Gamma})$ denote any optimal solutions to the optimization problems (40) and (41) defining the two-step procedure. We again define the error matrices $\widehat{\Delta}^{\Theta}=\widehat{\Theta}-\Theta^{\star}$ and $\widehat{\Delta}^{\Gamma}=\widehat{\Gamma}-\Gamma^{\star}$, the matrices $\widehat{\Delta}_{\mathbb{M}}^{\Gamma}:=\Pi_{\mathbb{M}}\left(\widehat{\Delta}^{\Gamma}\right)$ and $\widehat{\Delta}_{\mathbb{M}^{\perp}}^{\Gamma}=\Pi_{\mathbb{M} \perp}\left(\widehat{\Delta}^{\Gamma}\right)$, and the matrices $\Gamma^{\star} \mathbb{M}$ and $\Gamma^{\star} \mathbb{M}^{\perp}$ as previously defined in the proof of Theorem 1 .

Our proof of Proposition 1 is based on two lemmas, of which the first provides control on the error $\widehat{\Delta}^{\Gamma}$ in estimating the sparse component.

Lemma 3. Under the assumptions of Proposition 1, for any subset $S$ of matrix indices of cardinality at most $s$, the sparse error $\widehat{\Delta}^{\Gamma}$ in any solution of the convex program (40) satisfies the bound

$$
\left\|\widehat{\Delta}^{\Gamma}\right\|_{\mathrm{F}}^{2} \leq c_{1} \mu_{d}^{2}\left\{s+\frac{1}{\mu_{d}} \sum_{(j, k) \notin S}\left|\Gamma^{\star}{ }_{j k}\right|\right\} .
$$

Proof. Since $\widehat{\Gamma}$ and $\Gamma^{\star}$ are optimal and feasible (respectively) for the convex program (40), we have

$$
\frac{1}{2}\|\widehat{\Gamma}-Y\|_{\mathrm{F}}^{2}+\mu_{d}\|\widehat{\Gamma}\|_{1} \leq \frac{1}{2}\left\|\Theta^{\star}+W\right\|_{\mathrm{F}}^{2}+\mu_{d}\left\|\Gamma^{\star}\right\|_{1}
$$

Re-writing this inequality in terms of the error $\widehat{\Delta}^{\Gamma}$ and re-arranging terms yields

$$
\frac{1}{2}\left\|\widehat{\Delta}^{\Gamma}\right\|_{\mathrm{F}}^{2} \leq\left|\left\langle\left\langle\widehat{\Delta}^{\Gamma}, W+\Theta^{\star}\right\rangle\right\rangle\right|+\mu_{d}\left\|\Gamma^{\star}\right\|_{1}-\mu_{d}\left\|\Gamma^{\star}+\widehat{\Delta}^{\Gamma}\right\|_{1} .
$$

By decomposability of the $\ell_{1}$-norm, we obtain

$$
\begin{aligned}
\frac{1}{2}\left\|\widehat{\Delta}^{\Gamma}\right\|_{\mathrm{F}}^{2} & \leq\left|\left\langle\left\langle\widehat{\Delta}^{\Gamma}, W+\Theta^{\star}\right\rangle\right\rangle\right|+\mu_{d}\left\{\left\|\Gamma_{S}^{*}\right\|_{1}+\left\|\Gamma_{S^{c}}^{*}\right\|_{1}-\left\|\Gamma_{S}^{*}+\widehat{\Delta}_{S}^{\Gamma}\right\|_{1}-\left\|\Gamma_{S^{c}}^{*}+\widehat{\Delta}_{S^{c}}^{\Gamma_{1}}\right\|_{1}\right\} \\
& \leq\left|\left\langle\left\langle\widehat{\Delta}^{\Gamma}, W+\Theta^{\star}\right\rangle\right\rangle\right|+\mu_{d}\left\{2\left\|\Gamma_{S^{c}}^{*}\right\|_{1}+\left\|\widehat{\Delta}_{S}^{\Gamma}\right\|_{1}-\left\|\widehat{\Delta}_{S^{c}}^{\Gamma}\right\|_{1}\right\},
\end{aligned}
$$

where the second step is based on two applications of the triangle inequality. Now by applying Hölder's inequality and the triangle inequality to the first term on the right-hand side, we obtain

$$
\begin{aligned}
\frac{1}{2}\left\|\widehat{\Delta}^{\Gamma}\right\|_{\mathrm{F}}^{2} \leq & \left\|\widehat{\Delta}^{\Gamma}\right\|_{1}\left[\|W\|_{\infty}+\left\|\Theta^{\star}\right\|_{\infty}\right]+\mu_{d}\left\{2\left\|\Gamma_{S^{c}}^{*}\right\|_{1}+\left\|\widehat{\Delta}_{S}^{\Gamma}\right\|_{1}-\left\|\widehat{\Delta}_{S^{c}}^{\Gamma}\right\|_{1}\right\} \\
= & \left\|\widehat{\Delta}_{S}^{\Gamma}\right\|_{1}\left\{\|W\|_{\infty}+\left\|\Theta^{\star}\right\|_{\infty}+\mu_{d}\right\}+\left\|\widehat{\Delta}_{S^{c}}^{\Gamma}\right\|_{1}\left\{\|W\|_{\infty}+\left\|\Theta^{\star}\right\|_{\infty}-\mu_{d}\right\}+2 \mu_{d}\left\|\Gamma_{S^{c}}^{*}\right\|_{1} \\
\leq & 2 \mu_{d}\left\|\widehat{\Delta}_{S}^{\Gamma}\right\|_{1}+2 \mu_{d}\left\|\Gamma_{S^{c}}^{*}\right\|_{1}, \\
& \quad \text { imsart-aos ver. 2007/12/10 file: A0S_supp.tex date: June 5, } 2012
\end{aligned}
$$


where the final inequality follows from our stated choice (42) of the regularization parameter $\mu_{d}$. Since $\left\|\widehat{\Delta}_{S}^{\Gamma}\right\|_{1} \leq \sqrt{s}\left\|\widehat{\Delta}_{S}\right\|_{\mathrm{F}} \leq \sqrt{s}\left\|\widehat{\Delta}^{\Gamma}\right\|_{\mathrm{F}}$, the claim (59) follows with some algebra.

Our second lemma provides a bound on the low-rank error $\widehat{\Delta}^{\Theta}$ in terms of the sparse matrix error $\widehat{\Delta}^{\Gamma}$.

Lemma 4. If in addition to the conditions of Proposition 1, the sparse error matrix is bounded as $\left\|\widehat{\Delta}^{\Gamma}\right\|_{\mathrm{F}} \leq \delta$, then the low-rank error matrix is bounded as

$$
\left\|\widehat{\Delta}^{\Theta}\right\|_{\mathrm{F}}^{2} \leq c_{1} \lambda_{d}^{2}\left\{r+\frac{1}{\lambda_{d}} \sum_{j=r+1}^{d} \sigma_{j}\left(\Theta^{\star}\right)\right\}+c_{2} \delta^{2} .
$$

As the proof of this lemma is somewhat more involved, we defer it to Appendix 11. Finally, combining the low-rank bound (60) with the sparse bound (59) from Lemma 3 yields the claim of Proposition 1

7.5. Proof of Corollary [6. For this corollary, we have $\mathcal{R}(\cdot)=\|\cdot\|_{2,1}$ and $\mathcal{R}^{*}(\cdot)=\|\cdot\|_{2, \infty}$. In order to establish the claim, we need to show that the conditions of Corollary 5 on the regularization pair $\left(\lambda_{d}, \mu_{d}\right)$ hold with high probability. The setting of $\lambda_{d}$ is the same as Corollary 2, and is valid by our earlier argument. Hence, in order to complete the proof, it remains to establish an upper bound on $\|W\|_{2, \infty}$.

Let $W_{k}$ be the $k^{t h}$ column of the matrix. Noting that the function $W_{k} \mapsto$ $\left\|W_{k}\right\|_{2}$ is Lipschitz, by concentration of measure for Gaussian Lipschitz functions [18], we have

$$
\mathbb{P}\left[\left\|W_{k}\right\|_{2} \geq \mathbb{E}\left\|W_{k}\right\|_{2}+t\right] \leq \exp \left(-\frac{t^{2} d_{1} d_{2}}{2 \nu^{2}}\right) \quad \text { for all } t>0 .
$$

Using the Gaussianity of $W_{k}$, we have $\mathbb{E}\left\|W_{k}\right\|_{2} \leq \frac{\nu}{\sqrt{d_{1} d_{2}}} \sqrt{d_{1}}=\frac{\nu}{\sqrt{d_{2}}}$. Applying union bound over all $d_{2}$ columns, we conclude that with probability greater than $1-\exp \left(-\frac{t^{2} d_{1} d_{2}}{2 \nu^{2}}+\log d_{2}\right)$, we have $\max _{k}\left\|W_{k}\right\|_{2} \leq \frac{\nu}{\sqrt{d_{2}}}+t$. Setting $t=4 \nu \sqrt{\frac{\log d_{2}}{d_{1} d_{2}}}$ yields

$$
\mathbb{P}\left[\|W\|_{2, \infty} \geq \frac{\nu}{\sqrt{d_{2}}}+4 \nu \sqrt{\frac{\log d_{2}}{d_{1} d_{2}}}\right] \leq \exp \left(-3 \log d_{2}\right),
$$

from which the claim follows.

As before, a sharper bound (with $\log d_{2}$ replaced by $\log \left(d_{2} / s\right)$ ) can be obtained by a refined argument; we refer the reader to Appendix 10.2 for the details. 
7.6. Proof of Corollary 7 . For this model, the noise matrix takes the form $W:=\frac{1}{n} \sum_{i=1}^{n} U_{i} U_{i}^{T}-\Theta^{\star}$, where $U_{i} \sim N\left(0, \Theta^{\star}\right)$. Since $\Theta^{\star}$ is positive semidefinite with rank at most $r$, we can write

$$
W=Q\left\{\frac{1}{n} Z_{i} Z_{i}^{T}-I_{r \times r}\right\} Q^{T},
$$

where the matrix $Q \in \mathbb{R}^{d \times r}$ satisfies the relationship $\Theta^{\star}=Q Q^{T}$, and $Z_{i} \sim N\left(0, I_{r \times r}\right)$ is standard Gaussian in dimension $r$. Consequently, by known results on singular values of Wishart matrices [11, we have $\|W\|_{\text {op }} \leq$ $\sqrt{8}\left\|\Theta^{\star}\right\|_{\text {op }} \sqrt{\frac{r}{n}}$ with high probability, showing that the specified choice of $\lambda_{d}$ is valid. It remains to bound the quantity $\|W\|_{2, \infty}$. By known matrix norm bounds [15], we have $\|W\|_{2, \infty} \leq\|W\|_{\text {op }}$, so that the claim follows by the previous argument.

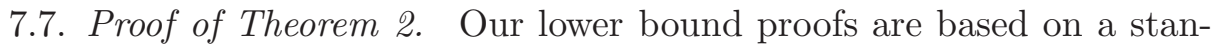
dard reduction [14, 33, 32] from estimation to a multiway hypothesis testing problem over a packing set of matrix pairs. In particular, given a collection $\left\{\left(\Theta^{j}, \Gamma^{j}\right), j=1,2, \ldots, M\right\}$ of matrix pairs contained in some family $\mathcal{F}$, we say that it forms a $\delta$-packing in Frobenius norm if, for all distinct pairs $i, j \in\{1,2, \ldots, M\}$, we have

$$
\left\|\Theta^{i}-\Theta^{j}\right\|_{F}^{2}+\left\|\Gamma^{i}-\Gamma^{j}\right\|_{F}^{2} \geq \delta^{2} .
$$

Given such a packing set, it is a straightforward consequence of Fano's inequality that the minimax error over $\mathcal{F}$ satisfies the lower bound

$$
\mathbb{P}\left[\mathfrak{M}(\mathcal{F}) \geq \frac{\delta^{2}}{8}\right] \geq 1-\frac{I(Y ; J)+\log 2}{\log M},
$$

where $I(Y ; J)$ is the mutual information between the observation matrix $Y \in \mathbb{R}^{d_{1} \times d_{2}}$, and $J$ is an index uniformly distributed over $\{1,2, \ldots, M\}$. In order to obtain different components of our bound, we make different choices of the packing set, and use different bounding techniques for the mutual information.

7.7.1. Lower bounds for elementwise sparsity. We begin by proving the lower bound (50) for matrix decompositions over the family $\mathcal{F}_{\mathrm{sp}}(r, s, \alpha)$.

Packing for radius of non-identifiability. Let us first establish the lower bound involving the radius of non-identifiability, namely the term scaling as $\frac{\alpha^{2} s}{d_{1} d_{2}}$ in the case of $s$-sparsity for $\Theta^{\star}$. Recall from Example 6 the "bad" matrix (33), which we denote here by $B^{*}$. By construction, we have $\left\|B^{*}\right\|_{F}^{2}=$ 
$\frac{\alpha^{2} s}{d_{1} d_{2}}$. Using this matrix, we construct a very simple packing set with $M=4$ matrix pairs $(\Theta, \Gamma)$ :

$$
\left\{\left(B^{*},-B^{*}\right),\left(-B^{*}, B^{*}\right),\left(\frac{1}{\sqrt{2}} B^{*},-\frac{1}{\sqrt{2}} B^{*}\right),(0,0)\right\}
$$

Each one of these matrix pairs $(\Theta, \Gamma)$ belongs to the set $\mathcal{F}_{\mathrm{sp}}(1, s, \alpha)$, so it can be used to establish a lower bound over this set. (Moreover, it also yields a lower bound over the sets $\mathcal{F}_{\mathrm{sp}}(r, s, \alpha)$ for $r>1$, since they are supersets.) It can also be verified that for any two distinct pairs of matrices in the set (62), they differ in squared Frobenius norm by at least $\delta^{2}=\frac{1}{2}\left\|B^{*}\right\|_{F}^{2}=$ $\frac{1}{2} \frac{\alpha^{2} s}{d_{1} d_{2}}$. Let $J$ be a random index uniformly distributed over the four possible models in our packing set (62). By construction, for any matrix pair $(\Theta, \Gamma)$ in the packing set, we have $\Theta+\Gamma=0$. Consequently, for any one of these models, the observation matrix $Y$ is simply equal to pure noise $W$, and hence $I(Y ; J)=0$. Putting together the pieces, the Fano bound (61) implies that

$$
\mathbb{P}\left[\mathfrak{M}\left(\mathcal{F}_{\mathrm{sp}}(1, s, \alpha)\right) \geq \frac{1}{16} \frac{\alpha^{2} s}{d_{1} d_{2}}\right] \geq 1-\frac{I(Y ; J)+\log 2}{\log 4}=\frac{1}{2} .
$$

Packing for estimation error:. We now describe the construction of a packing set for lower bounding the estimation error. In this case, our construction is more subtle, based on the the Cartesian product of two components, one for the low rank matrices, and the other for the sparse matrices. For the low rank component, we re-state a slightly modified form (adapted to the setting of non-square matrices) of Lemma 2 from the paper [23]:

Lemma 5. For $d_{1}, d_{2} \geq 10$, a tolerance $\delta>0$, and for each $r=1,2, \ldots, d$, there exists a set of $d_{1} \times d_{2}$-dimensional matrices $\left\{\Theta^{1}, \ldots, \Theta^{M}\right\}$ with cardinality $M \geq \frac{1}{4} \exp \left(\frac{r d_{1}}{256}+\frac{r d_{2}}{256}\right)$ such that each matrix has rank $r$, and moreover

$$
\begin{aligned}
\left\|\Theta^{\ell}\right\|_{F}^{2} & =\delta^{2} \quad \text { for all } \ell=1,2, \ldots, M, \\
\left\|\Theta^{\ell}-\Theta^{k}\right\|_{F}^{2} & \geq \delta^{2} \quad \text { for all } \ell \neq k, \\
\left\|\Theta^{\ell}\right\|_{\infty} & \leq \delta \sqrt{\frac{32 \log \left(d_{1} d_{2}\right)}{d_{1} d_{2}}} \quad \text { for all } \ell=1,2, \ldots, M .
\end{aligned}
$$

Consequently, as long as $\delta \leq 1$, we are guaranteed that the matrices $\Theta^{\ell}$ belong to the set $\mathcal{F}_{\mathrm{sp}}(r, s, \alpha)$ for all $\alpha \geq 32 \sqrt{\log \left(d_{1} d_{2}\right)}$.

As for the sparse matrices, the following result is a modification, so as to apply to the matrix setting of interest here, of Lemma 5 from the paper [25]:

imsart-aos ver. 2007/12/10 file: AOS_supp.tex date: June 5, 2012 
Lemma 6 (Sparse matrix packing). For any $\delta>0$, and for each integer $s<d_{1} d_{2}$, there exists a set of matrices $\left\{\Gamma^{1}, \ldots, \Gamma^{N}\right\}$ with cardinality $N \geq$ $\exp \left(\frac{s}{2} \log \frac{d_{1} d_{2}-s}{s / 2}\right)$ such that

$$
\begin{aligned}
\left\|\Gamma^{j}-\Gamma^{k}\right\|_{\mathrm{F}}^{2} & \geq \delta^{2}, \quad \text { and } \\
\left\|\Gamma^{j}\right\|_{\mathrm{F}}^{2} & \leq 8 \delta^{2},
\end{aligned}
$$

and such that each $\Gamma^{j}$ has at most s non-zero entries.

We now have the necessary ingredients to prove the lower bound (50). By combining Lemmas 5 and [6, we conclude that there exists a set of matrices with cardinality

$$
M N \geq \frac{1}{4} \exp \left\{\frac{s}{2} \log \frac{d_{1} d_{2}-s}{s / 2}+\frac{r d_{1}}{256}+\frac{r d_{2}}{256}\right\}
$$

such that

$\left\|\left(\Theta^{\ell}, \Gamma^{k}\right)-\left(\Theta^{\ell^{\prime}}, \Gamma^{k^{\prime}}\right)\right\|_{\mathrm{F}}^{2} \geq \delta^{2} \quad$ for all pairs such that $\ell \neq \ell^{\prime}$ or $k \neq k^{\prime}$, and

$$
\left\|\left(\Theta^{\ell}, \Gamma^{k}\right)\right\|_{\mathrm{F}}^{2} \leq 9 \delta^{2} \quad \text { for all }(\ell, k) .
$$

Let $\mathbb{P}^{\ell, k}$ denote the distribution of the observation matrix $Y$ when $\Theta^{\ell}$ and $\Gamma^{k}$ are the underlying parameters. We apply the Fano construction over the class of $M N$ such distributions, thereby obtaining that in order to show that the minimax error is lower bounded by $c_{0} \delta^{2}$ (for some universal constant $c_{0}>0$ ), it suffices to show that

$$
\frac{\frac{1}{\left(\begin{array}{c}
M N \\
2
\end{array}\right)} \sum_{(\ell, k) \neq\left(\ell^{\prime}, k^{\prime}\right)} \mathbb{D}\left(\mathbb{P}^{\ell, k} \| \mathbb{P}^{\ell^{\prime}, k^{\prime}}\right)+\log 2}{\log (M N)} \leq \frac{1}{2},
$$

where $\mathbb{D}\left(\mathbb{P}^{\ell, k} \| \mathbb{P}^{\ell^{\prime}, k^{\prime}}\right)$ denotes the Kullback-Leibler divergence between the distributions $\mathbb{P}^{\ell, k}$ and $\mathbb{P}^{\ell^{\prime}, k^{\prime}}$. Given the assumption of Gaussian noise with variance $\nu^{2} /\left(d_{1} d_{2}\right)$, we have

$$
\mathbb{D}\left(\mathbb{P}^{j} \| \mathbb{P}^{k}\right)=\frac{d_{1} d_{2}}{2 \nu^{2}}\left\|\left(\Theta^{\ell}, \Gamma^{k}\right)-\left(\Theta^{\ell^{\prime}}, \Gamma^{k^{\prime}}\right)\right\|_{\mathrm{F}}^{2} \stackrel{(i)}{\leq} \frac{18 d_{1} d_{2} \delta^{2}}{\nu^{2}},
$$

where the bound (i) follows from the condition (66b). Combined with lower bound (65), we see that it suffices to choose $\delta$ such that

$$
\begin{gathered}
\frac{\frac{18 d_{1} d_{2} \delta^{2}}{\nu^{2}}+\log 2}{\log \frac{1}{4}+\left\{\frac{s}{2} \log \frac{d_{1} d_{2}-s}{s / 2}+\frac{r d_{1}}{256}+\frac{r d_{2}}{256}\right\}} \leq \frac{1}{2} . \\
\text { imsart-aos ver. } 2007 / 12 / 10 \text { file: A0S_supp.tex date: June 5, } 2012
\end{gathered}
$$


For $d_{1}, d_{2}$ larger than a finite constant (to exclude degenerate cases), we see that the choice

$$
\delta^{2}=c_{0} \nu^{2}\left\{\frac{r}{d_{1}}+\frac{r}{d_{2}}+\frac{s \log \frac{d_{1} d_{2}-s}{s / 2}}{d_{1} d_{2}}\right\},
$$

for a suitably small constant $c_{0}>0$ is sufficient, thereby establishing the lower bound (50).

7.7.2. Lower bounds for columnwise sparsity. The lower bound (51) for columnwise follows from a similar argument. The only modifications are in the packing sets.

Packing for radius of non-identifiability. In order to establish a lower bound of order $\frac{\alpha^{2} s}{d_{2}}$, recall the "bad" matrix (45) from Example 8, which we denote by $B^{*}$. By construction, it has squared Frobenius norm $\left\|B^{*}\right\|_{F}^{2}=\frac{\alpha^{2} s}{d_{2}}$. We use it to form the packing set

$$
\left\{\left(B^{*},-B^{*}\right),\left(-B^{*}, B^{*}\right),\left(\frac{1}{\sqrt{2}} B^{*},-\frac{1}{\sqrt{2}} B^{*}\right),(0,0)\right\}
$$

Each one of these matrix pairs $(\Theta, \Gamma)$ belongs to the set $\mathcal{F}_{\text {col }}(1, s, \alpha)$, so it can be used to establish a lower bound over this set. (Moreover, it also yields a lower bound over the sets $\mathcal{F}_{\text {col }}(r, s, \alpha)$ for $r>1$, since they are supersets.) It can also be verified that for any two distinct pairs of matrices in the set (69), they differ in squared Frobenius norm by at least $\delta^{2}=\frac{1}{2}\left\|B^{*}\right\|_{F}^{2}=\frac{1}{2} \frac{\alpha^{2} s}{d_{2}}$. Consequently, the same argument as before shows that

$$
\mathbb{P}\left[\mathfrak{M}\left(\mathcal{F}_{\mathrm{col}}(1, s, \alpha)\right) \geq \frac{1}{16} \frac{\alpha^{2} s}{d_{2}}\right] \geq 1-\frac{I(Y ; J)+\log 2}{\log 4}=\frac{1}{2} .
$$

Packing for estimation error:. We now describe packings for the estimation error terms. For the low-rank packing set, we need to ensure that the $(2, \infty)$ norm is controlled. From the bound (63c), we have the guarantee

$$
\left\|\Theta^{\ell}\right\|_{2, \infty} \leq \delta \sqrt{\frac{32 \log \left(d_{1} d_{2}\right)}{d_{2}}} \text { for all } \ell=1,2, \ldots, M,
$$

so that, as long as $\delta \leq 1$, the matrices $\Theta^{\ell}$ belong to the set $\mathcal{F}_{\text {col }}(r, s, \alpha)$ for all $\alpha \geq 32 \sqrt{\log \left(d_{1} d_{2}\right)}$.

The following lemma characterizes a suitable packing set for the columnwise sparse component: 
Lemma 7 (Columnwise sparse matrix packing). For all $d_{2} \geq 10$ and integers $s$ in the set $\left\{1,2, \ldots, d_{2}-1\right\}$, there exists a family $d_{1} \times d_{2}$ matrices $\left\{\Gamma^{k}, k=1,2, \ldots N\right\}$ with cardinality

$$
N \geq \exp \left(\frac{s}{8} \log \frac{d_{2}-s}{s / 2}+\frac{s d_{1}}{8}\right)
$$

satisfying the inequalities

$$
\begin{aligned}
\left\|\Gamma^{j}-\Gamma^{k}\right\|_{\mathrm{F}}^{2} & \geq \delta^{2}, \quad \text { for all } j \neq k, \text { and } \\
\left\|\Gamma^{j}\right\|_{\mathrm{F}}^{2} & \leq 64 \delta^{2},
\end{aligned}
$$

and such that each $\Gamma^{j}$ has at most s non-zero columns.

This claim follows by suitably adapting Lemma 5(b) in the paper by Raskutti et al. 26] on minimax rates for kernel classes. In particular, we view column $j$ of a matrix $\Gamma$ as defining a linear function in dimension $\mathbb{R}^{d_{1}}$; for each $j=1,2, \ldots, d_{1}$, this defines a Hilbert space $\mathcal{H}_{j}$ of functions. By known results on metric entropy of Euclidean balls [19], this function class has logarithmic metric entropy, so that part (b) of the above lemma applies, and yields the stated result.

Using this lemma and the packing set for the low-rank component and following through the Fano construction yields the claimed lower bound (50) on the minimax error for the class $\mathcal{F}_{\text {col }}(r, s, \alpha)$, which completes the proof of Theorem 2 .

8. Proof of Lemma 1. The decomposition described in part (a) was established by Recht et al. [27], so that it remains to prove part (b). With the appropriate definitions, part (b) can be recovered by exploiting Lemma 1 from Negahban et al. 21. Their lemma applies to optimization problems of the general form

$$
\min _{\theta \in \mathbb{R}^{p}}\left\{\mathcal{L}(\theta)+\gamma_{n} r(\theta)\right\}
$$

where $\mathcal{L}$ is a loss function on the parameter space, and $r$ is norm-based regularizer that satisfies a property known as decomposability. The elementwise $\ell_{1}$-norm as well as the nuclear norm are both instances of decomposable regularizers. Their lemma requires that the regularization parameter $\gamma_{n}$ be chosen such that $\gamma_{n} \geq 2 r^{*}\left(\nabla \mathcal{L}\left(\theta^{*}\right)\right)$, where $r^{*}$ is the dual norm, and $\nabla \mathcal{L}\left(\theta^{*}\right)$ is the gradient of the loss evaluated at the true parameter.

We now discuss how this lemma can be applied in our special case. Here the relevant parameters are of the form $\theta=(\Theta, \Gamma)$, and the loss function is 
given by

$$
\mathcal{L}(\Theta, \Gamma)=\frac{1}{2}\|Y-(\Theta+\Gamma)\|_{\mathrm{F}}^{2} .
$$

The sample size $n=d^{2}$, since we make one observation for each entry of the matrix. On the other hand, the regularizer is given by the function

$$
r(\theta)=\mathcal{Q}(\Theta, \Gamma):=\|\Theta\|_{\mathrm{N}}+\frac{\mu_{d}}{\lambda_{d}} \mathcal{R}(\Gamma),
$$

coupled with the regularization parameter $\gamma_{n}=\lambda_{d}$. By assumption, the regularizer $\mathcal{R}$ is decomposable, and as shown in the paper [21], the nuclear norm is also decomposable. Since $\mathcal{Q}$ is simply a sum of these decomposable regularizers over separate matrices, it is also decomposable.

It remains to compute the gradient $\nabla \mathcal{L}\left(\Theta^{\star}, \Gamma^{\star}\right)$, and evaluate the dual norm. A straightforward calculation yields that $\nabla \mathcal{L}\left(\Theta^{\star}, \Gamma^{\star}\right)=\left[\begin{array}{ll}W & W\end{array}\right]^{T}$. In addition, it can be verified by standard properties of dual norms

$$
\mathcal{Q}^{*}(U, V)=\|U\|_{\mathrm{op}}+\frac{\lambda_{d}}{\mu_{d}} \mathcal{R}^{*}(V) .
$$

Thus, it suffices to choose the regularization parameter such that

$$
\lambda_{d} \geq 2 \mathcal{Q}^{*}(W, W)=2\|W\|_{\mathrm{op}}+\frac{2 \lambda_{d}}{\mu_{d}} \mathcal{R}^{*}(W) .
$$

Given our condition (52), we have

$$
2\|W\|_{\text {op }}+\frac{2 \lambda_{d}}{\mu_{d}} \mathcal{R}^{*}(W) \leq 2\|W\|_{\text {op }}+\frac{\lambda_{d}}{2},
$$

meaning that it suffices to have $\lambda_{d} \geq 4\|W\|_{\text {op }}$, as stated in the second part of condition (52).

9. Proof of Lemma 2, By the RSC condition (22), we have

$\frac{1}{2}\left\|\mathfrak{X}\left(\widehat{\Delta}^{\Theta}+\widehat{\Delta}^{\Gamma}\right)\right\|_{\mathrm{F}}^{2}-\frac{\gamma}{2}\left\|\widehat{\Delta}^{\Theta}+\widehat{\Delta}^{\Gamma}\right\|_{\mathrm{F}}^{2} \geq-\tau_{n} \Phi^{2}\left(\widehat{\Delta}^{\Theta}+\widehat{\Delta}^{\Gamma}\right) \geq-\tau_{n} \mathcal{Q}^{2}\left(\widehat{\Delta}^{\Theta}, \widehat{\Delta}^{\Gamma}\right)$,

where the second inequality follows by the definitions (20) and (21) of $\mathcal{Q}$ and $\Phi$ respectively. We now derive a lower bound on $\left\|\widehat{\Delta}^{\Theta}+\widehat{\Delta}^{\Gamma}\right\|_{F}$, and an upper bound on $\mathcal{Q}^{2}\left(\widehat{\Delta}^{\Theta}, \widehat{\Delta}^{\Gamma}\right)$. Beginning with the former term, observe that

$$
\begin{aligned}
& \frac{\gamma}{2}\left(\left\|\widehat{\Delta}^{\Theta}\right\|_{F}^{2}+\left\|\widehat{\Delta}^{\Gamma}\right\|_{F}^{2}\right)-\frac{\gamma}{2}\left\|\widehat{\Delta}^{\Theta}+\widehat{\Delta}^{\Gamma}\right\|_{F}^{2}=-\gamma\left\langle\left\langle\widehat{\Delta}^{\Theta}, \widehat{\Delta}^{\Gamma}\right\rangle\right\rangle \\
& \quad \text { imsart-aos ver. } 2007 / 12 / 10 \text { file: AOS_supp.tex date: June 5, } 2012
\end{aligned}
$$


so that it suffices to upper bound $\gamma\left|\left\langle\left\langle\widehat{\Delta}^{\Theta}, \widehat{\Delta}^{\Gamma}\right\rangle\right\rangle\right|$. By the duality of the pair $\left(\mathcal{R}, \mathcal{R}^{*}\right)$, we have

$$
\gamma\left|\left\langle\widehat{\Delta}^{\Theta}, \widehat{\Delta}^{\Gamma}\right\rangle\right\rangle \mid \leq \gamma \mathcal{R}^{*}\left(\widehat{\Delta}^{\Theta}\right) \mathcal{R}\left(\widehat{\Delta}^{\Gamma}\right)
$$

Now since $\widehat{\Theta}$ and $\Theta^{\star}$ are both feasible for the program (17) and recalling that $\widehat{\Delta}^{\Theta}=\widehat{\Theta}-\Theta^{\star}$, an application of triangle inequality yields

$$
\gamma \mathcal{R}^{*}\left(\widehat{\Delta}^{\Theta}\right) \leq \gamma\left\{\mathcal{R}^{*}(\widehat{\Theta})+\mathcal{R}^{*}\left(\Theta^{\star}\right)\right\} \leq \frac{2 \alpha \gamma}{\kappa_{d}} \stackrel{(i)}{\leq} \frac{\mu_{d}}{2}
$$

where inequality (i) follows from our choice of $\mu_{d}$. Putting together the pieces, we have shown that

$$
\frac{\gamma}{2}\left\|\widehat{\Delta}^{\Theta}+\widehat{\Delta}^{\Gamma}\right\|_{\mathrm{F}}^{2} \geq \frac{\gamma}{2}\left(\left\|\widehat{\Delta}^{\Theta}\right\|_{F}^{2}+\left\|\widehat{\Delta}^{\Gamma}\right\|_{F}^{2}\right)-\frac{\mu_{d}}{2} \mathcal{R}\left(\widehat{\Delta}^{\Gamma}\right) .
$$

Since the quantity $\lambda_{d}\left\|\widehat{\Delta}^{\Theta}\right\|_{\mathrm{N}} \geq 0$, we can write

$$
\begin{aligned}
\frac{\gamma}{2}\left\|\widehat{\Delta}^{\Theta}+\widehat{\Delta}^{\Gamma}\right\|_{\mathrm{F}}^{2} & \geq \frac{\gamma}{2}\left(\left\|\widehat{\Delta}^{\Theta}\right\|_{F}^{2}+\left\|\widehat{\Delta}^{\Gamma}\right\|_{F}^{2}\right)-\frac{\mu_{d}}{2} \mathcal{R}\left(\widehat{\Delta}^{\Gamma}\right)-\frac{\lambda_{d}}{2}\left\|\widehat{\Delta}^{\Theta}\right\|_{\mathrm{N}} \\
& =\frac{\gamma}{2}\left(\left\|\widehat{\Delta}^{\Theta}\right\|_{F}^{2}+\left\|\widehat{\Delta}^{\Gamma}\right\|_{F}^{2}\right)-\frac{\lambda_{d}}{2} \mathcal{Q}\left(\widehat{\Delta}^{\Theta}, \widehat{\Delta}^{\Gamma}\right),
\end{aligned}
$$

where the latter equality follows by the definition (20) of $\mathcal{Q}$.

Next we turn to the upper bound on $\mathcal{Q}\left(\widehat{\Delta}^{\Theta}, \widehat{\Delta}^{\Gamma}\right)$. By the triangle inequality, we have

$$
\mathcal{Q}\left(\widehat{\Delta}^{\Theta}, \widehat{\Delta}^{\Gamma}\right) \leq \mathcal{Q}\left(\widehat{\Delta}_{A}^{\Theta}, \widehat{\Delta}_{\mathbb{M}}^{\Gamma}\right)+\mathcal{Q}\left(\widehat{\Delta}_{B}^{\Theta}, \widehat{\Delta}_{\mathbb{M}^{\perp}}^{\Gamma}\right)
$$

Furthermore, substituting in equation (53) into the above equation yields

$$
\mathcal{Q}\left(\widehat{\Delta}^{\Theta}, \widehat{\Delta}^{\Gamma}\right) \leq 4 \mathcal{Q}\left(\widehat{\Delta}_{A}^{\Theta}, \widehat{\Delta}_{\mathbb{M}}^{\Gamma}\right)+4\left\{\sum_{j=r+1}^{d} \sigma_{j}\left(\Theta^{\star}\right)+\frac{\mu_{d}}{\lambda_{d}} \mathcal{R}\left(\Gamma^{\star}{ }_{\mathbb{M}^{\perp}}\right)\right\}
$$

Since $\widehat{\Delta}_{A}^{\Theta}$ has rank at most $2 r$ and $\widehat{\Delta}_{\mathbb{M}}^{\Gamma}$ belongs to the model space $\mathbb{M}$, we have

$$
\begin{aligned}
\lambda_{d} \mathcal{Q}\left(\widehat{\Delta}_{A}^{\Theta}, \widehat{\Delta}_{\mathbb{M}}^{\Gamma}\right) & \leq \sqrt{2 r} \lambda_{d}\left\|\widehat{\Delta}_{A}^{\Theta}\right\|_{\mathrm{F}}+\Psi(\mathbb{M}) \mu_{d}\left\|\widehat{\Delta}_{\mathbb{M}}^{\Gamma}\right\|_{\mathrm{F}} \\
& \leq \sqrt{2 r} \lambda_{d}\left\|\widehat{\Delta}^{\Theta}\right\|_{\mathrm{F}}+\Psi(\mathbb{M}) \mu_{d}\left\|\widehat{\Delta}^{\Gamma}\right\|_{\mathrm{F}} .
\end{aligned}
$$

The claim then follows by substituting the above equation into equation (73), and then substituting the result into the earlier inequality (72). 
10. Refinement of achievability results. In this appendix, we provide refined arguments that yield sharpened forms of Corollaries 2 and 6 , These refinements yield achievable bounds that match the minimax lower bounds in Theorem 2 up to constant factors. We note that these refinements are significantly different only when the sparsity index s scales as $\Theta\left(d_{1} d_{2}\right)$ for Corollary 2, or as $\Theta\left(d_{2}\right)$ for Corollary 6 .

10.1. Refinement of Corollary 2, In the proof of Theorem 1, when specialized to the $\ell_{1}$-norm, the noise term $\left|\left\langle W, \widehat{\Delta}^{\Gamma}\right\rangle\right|$ is simply upper bounded by $\|W\|_{\infty}\left\|\widehat{\Delta}^{\Gamma}\right\|_{1}$. Here we use a more careful argument to control this noise term. Throughout the proof, we assume that the regularization parameter $\lambda_{d}$ is set in the usual way, whereas we choose

$$
\mu_{d}=16 \nu \sqrt{\frac{\log \frac{d_{1} d_{2}}{s}}{d_{1} d_{2}}}+\frac{4 \alpha}{\sqrt{d_{1} d_{2}}} .
$$

We split our analysis into two cases.

Case 1: First, suppose that $\left\|\widehat{\Delta}^{\Gamma}\right\|_{1} \leq \sqrt{s}\left\|\widehat{\Delta}^{\Gamma}\right\|_{\mathrm{F}}$. In this case, we have the upper bound

$$
\left|\left\langle W, \widehat{\Delta}^{\Gamma}\right\rangle\right| \leq \sup _{\substack{\|\Delta\|_{1} \leq \sqrt{s}\left\|\widehat{\Delta}^{\Gamma}\right\|_{F} \\\|\Delta\|_{F} \leq\|\| \widehat{\Delta}^{\Gamma} \|_{F}}}|\langle W, \Delta\rangle\rangle \mid=\left\|\widehat{\Delta}^{\Gamma}\right\|_{F} \underbrace{\sup _{\substack{\|\Delta\|_{1} \leq \sqrt{s} \\\|\Delta\|_{F} \leq 1}}|\langle W, \Delta\rangle\rangle \mid}_{Z(s)}
$$

It remains to upper bound the random variable $Z(s)$. Viewed as a function of $W$, it is a Lipschitz function with parameter $\frac{\nu}{\sqrt{d_{1} d_{2}}}$, so that

$$
\mathbb{P}[Z(s) \geq \mathbb{E}[Z(s)]+\delta] \leq \exp \left(-\frac{d_{1} d_{2} \delta^{2}}{2 \nu^{2}}\right) .
$$

Setting $\delta^{2}=\frac{4 s \nu^{2}}{d_{1} d_{2}} \log \left(\frac{d_{1} d_{2}}{s}\right)$, we have

$$
Z(s) \leq \mathbb{E}[Z(s)]+\frac{2 s \nu}{d_{1} d_{2}}\left[\log \left(\frac{d_{1} d_{2}}{s}\right)\right]
$$

with probability greater than $1-\exp \left(-2 s \log \left(\frac{d_{1} d_{2}}{s}\right)\right)$.

It remains to upper bound the expected value. In order to do so, we apply Theorem 5.1(ii) from Gordon et al. [13] with $\left(q_{0}, q_{1}\right)=(1,2), n=d_{1} d_{2}$ and $t=\sqrt{s}$, thereby obtaining

$$
\begin{aligned}
\mathbb{E}[Z(t)] \leq & c^{\prime} \frac{\nu}{\sqrt{d_{1} d_{2}}} \sqrt{s} \sqrt{2+\log \left(\frac{2 d_{1} d_{2}}{s}\right)} \leq c \frac{\nu}{\sqrt{d_{1} d_{2}}} \sqrt{s \log \left(\frac{d_{1} d_{2}}{s}\right)} . \\
& \text { imsart-aos ver. 2007/12/10 file: A0S_supp.tex date: June 5, } 2012
\end{aligned}
$$


With this bound, proceeding through the remainder of the proof yields the claimed rate.

Case 2:. Alternatively, we must have $\left\|\widehat{\Delta}^{\Gamma}\right\|_{1}>\sqrt{s}\left\|\widehat{\Delta}^{\Gamma}\right\|_{\mathrm{F}}$. In this case, we need to show that the stated choice (74) of $\mu_{d}$ satisfies $\mu_{d}\left\|\widehat{\Delta}^{\Gamma}\right\|_{1} \geq$ $2\left|\left\langle W, \widehat{\Delta}^{\Gamma}\right\rangle\right\rangle \mid$ with high probability. As can be seen from examining the proofs, this condition is sufficient to ensure that Lemma 11 and Lemma 2 all hold, as required for our analysis.

We have the upper bound

$$
\left.\left|\left\langle W, \widehat{\Delta}^{\Gamma}\right\rangle\right\rangle\left|\leq \sup _{\substack{\|\Delta\|_{1} \leq\left\|\widehat{\Delta}^{\Gamma}\right\|_{1} \\\|\Delta\|_{F} \leq\left\|\widehat{\Delta}^{\Gamma}\right\|_{F}}}\right|\langle W, \Delta\rangle\right\rangle \mid=\left\|\widehat{\Delta}^{\Gamma}\right\|_{F} Z\left(\frac{\left\|\widehat{\Delta}^{\Gamma}\right\|_{1}}{\left\|\widehat{\Delta}^{\Gamma}\right\|_{F}}\right),
$$

where for any radius $t>0$, we define the random variable

$$
Z(t):=\sup _{\substack{\|\Delta\|_{1} \leq t \\\|\Delta\|_{\mathrm{F}} \leq 1}}|\langle W, \Delta\rangle\rangle \mid
$$

For each fixed $t$, the same argument as before shows that $Z(t)$ is concentrated around its expectation, and Theorem 5.1(ii) from Gordon et al. [13] with $\left(q_{0}, q_{1}\right)=(1,2), n=d_{1} d_{2}$ yields

$$
\mathbb{E}[Z(t)] \leq c \frac{\nu}{\sqrt{d_{1} d_{2}}} t \sqrt{\log \left(\frac{d_{1} d_{2}}{t^{2}}\right)} .
$$

Setting $\delta^{2}=\frac{4 t^{2} \nu^{2}}{d_{1} d_{2}} \log \left(\frac{d_{1} d_{2}}{s}\right)$ in the concentration bound, we conclude that

$$
Z(t) \leq c^{\prime} t \frac{\nu}{\sqrt{d_{1} d_{2}}}\left\{\sqrt{\log \left(\frac{d_{1} d_{2}}{s}\right)}+\sqrt{\log \left(\frac{d_{1} d_{2}}{t^{2}}\right)}\right\}
$$

with high probability. A standard peeling argument (e.g., [30]) can be used to extend this bound to a uniform one over the choice of radii $t$, so that it applies to the random one $t=\frac{\left\|\widehat{\Delta}^{\Gamma}\right\|_{1}}{\left\|\widehat{\Delta}^{\Gamma}\right\|_{\mathrm{F}}}$ of interest. (The only changes in doing such a peeling are in constant terms.) We thus conclude that

$Z\left(\frac{\left\|\widehat{\Delta}^{\Gamma}\right\|_{1}}{\left\|\widehat{\Delta}^{\Gamma}\right\|_{\mathrm{F}}}\right) \leq c^{\prime} \frac{\left\|\widehat{\Delta}^{\Gamma}\right\|_{1}}{\left\|\widehat{\Delta}^{\Gamma}\right\|_{\mathrm{F}}} \frac{\nu}{\sqrt{d_{1} d_{2}}}\left\{\sqrt{\log \left(\frac{d_{1} d_{2}}{s}\right)}+\sqrt{\log \left(\frac{d_{1} d_{2}}{\left\|\widehat{\Delta}^{\Gamma}\right\|_{1}^{2} /\left\|\widehat{\Delta}^{\Gamma}\right\|_{\mathrm{F}}^{2}}\right)}\right\}$ 
with high probability. Since $\left\|\widehat{\Delta}^{\Gamma}\right\|_{1}>\sqrt{s}\left\|\widehat{\Delta}^{\Gamma}\right\|_{\mathrm{F}}$, we have $\frac{1}{\left\|\widehat{\Delta}^{\Gamma}\right\|_{1}^{2} /\left\|\widehat{\Delta}^{\Gamma}\right\|_{\mathrm{F}}^{2}} \leq \frac{1}{s}$, and hence

$$
\left|\left\langle\left\langle W, \widehat{\Delta}^{\Gamma}\right\rangle\right\rangle\right| \leq\left\|\widehat{\Delta}^{\Gamma}\right\|_{\mathrm{F}} Z\left(\frac{\left\|\widehat{\Delta}^{\Gamma}\right\|_{1}}{\left\|\widehat{\Delta}^{\Gamma}\right\|_{\mathrm{F}}}\right) \leq c^{\prime \prime}\left\|\widehat{\Delta}^{\Gamma}\right\|_{1} \frac{\nu}{\sqrt{d_{1} d_{2}}} \sqrt{\log \left(\frac{d_{1} d_{2}}{s}\right)}
$$

with high probability. With this bound, the remainder of the proof proceeds as before. In particular, the refined choice (74) of $\mu_{d}$ is adequate.

10.2. Refinement of Corollary [6. As in the refinement of Corollary [2] from Appendix 10.1, we need to be more careful in controlling the noise term $\left\langle\left\langle W, \widehat{\Delta}^{\Gamma}\right\rangle\right\rangle$. For this corollary, we make the refined choice of regularizer

$$
\mu_{d}=16 \nu \sqrt{\frac{1}{d_{2}}}+16 \nu \sqrt{\frac{\log \left(d_{2} / s\right)}{d_{1} d_{2}}}+\frac{4 \alpha}{\sqrt{d_{2}}}
$$

As in Appendix 10.1, we split our analysis into two cases.

Case 1: First, suppose that $\left\|\widehat{\Delta}^{\Gamma}\right\|_{2,1} \leq \sqrt{s}\left\|\widehat{\Delta}^{\Gamma}\right\|_{\mathrm{F}}$. In this case, we have

$$
\left|\left\langle\left\langle W, \widehat{\Delta}^{\Gamma}\right\rangle\right\rangle\right| \leq \sup _{\substack{\|\Delta\|_{2,1} \leq \sqrt{s}\left\|\widehat{\Delta}^{\Gamma}\right\|_{F} \\\|\Delta\|_{F} \leq\left\|\widehat{\Delta}^{\Gamma}\right\|_{F}}}|\langle W, \Delta\rangle|=\left\|\widehat{\Delta}^{\Gamma}\right\|_{F} \underbrace{\sup _{\substack{\|\Delta\|_{2,1} \leq \sqrt{s} \\\|\Delta\|_{F} \leq 1}}|\langle W, \Delta\rangle|}_{\widetilde{Z}(s)}
$$

The function $W \mapsto \widetilde{Z}(s)$ is a Lipschitz function with parameter $\frac{\nu}{\sqrt{d_{1} d_{2}}}$, so that by concentration of measure for Gaussian Lipschitz functions [18], it satisfies the upper tail bound

$$
\mathbb{P}[\widetilde{Z}(s) \geq \mathbb{E}[\widetilde{Z}(s)]+\delta] \leq \exp \left(-\frac{d_{1} d_{2} \delta^{2}}{2 \nu^{2}}\right) .
$$

Setting $\delta^{2}=\frac{4 s \nu^{2}}{d_{1} d_{2}} \log \left(\frac{d_{2}}{s}\right)$ yields

$$
\widetilde{Z}(s) \leq \mathbb{E}[\widetilde{Z}(s)]+2 \nu \sqrt{\frac{s \log \left(\frac{d_{2}}{s}\right)}{d_{1} d_{2}}}
$$

with probability greater than $1-\exp \left(-2 s \log \left(\frac{d_{2}}{s}\right)\right)$. 
It remains to upper bound the expectation. Applying the Cauchy-Schwarz inequality to each column, we have

$$
\begin{aligned}
\mathbb{E}[\widetilde{Z}(s)] & \leq \mathbb{E}\left[\sup _{\substack{\|\Delta\|_{2,1} \leq \sqrt{s} \\
\|\Delta\|_{\mathrm{F}} \leq 1}} \sum_{k=1}^{d_{2}}\left\|W_{k}\right\|_{2}\left\|\Delta_{k}\right\|_{2}\right] \\
& =\mathbb{E}\left[\sup _{\substack{\|\Delta\|_{2,1} \leq \sqrt{s} \\
\|\Delta\|_{\mathrm{F}} \leq 1}} \sum_{k=1}^{d_{2}}\left(\left\|W_{k}\right\|_{2}-\mathbb{E}\left[\left\|W_{k}\right\|_{2}\right]\right)\left\|\Delta_{k}\right\|_{2}\right]+\sup _{\|\Delta\|_{2,1} \leq \sqrt{s}}\left(\sum_{k=1}^{d}\left\|\Delta_{k}\right\|_{2}\right) \mathbb{E}\left[\left\|W_{1}\right\|_{2}\right] \\
& \leq \mathbb{E}[\sup _{\substack{\|\Delta\|_{2,1} \leq \sqrt{s} \\
\|\Delta\|_{\mathrm{F}} \leq 1}} \sum_{k=1}^{d_{2}} \underbrace{\left(\left\|W_{k}\right\|_{2}-\mathbb{E}\left[\left\|W_{k}\right\|_{2}\right]\right)}_{V_{k}}\left\|\Delta_{k}\right\|_{2}]+4 \nu \sqrt{\frac{s}{d_{2}}},
\end{aligned}
$$

using the fact that $\mathbb{E}\left[\left\|W_{1}\right\|_{2}\right] \leq \nu \sqrt{\frac{d_{1}}{d_{2} d_{2}}}=\frac{\nu}{\sqrt{d_{2}}}$.

Now the variable $V_{k}$ is zero-mean, and sub-Gaussian with parameter $\frac{\nu}{\sqrt{d_{1} d_{2}}}$, again using concentration of measure for Lipschitz functions of Gaussians [18]. Consequently, by setting $\delta_{k}=\left\|\Delta_{k}\right\|_{2}$, we can write

$$
\mathbb{E}[\widetilde{Z}(s)] \leq \mathbb{E}\left[\sup _{\substack{\|\delta\|_{1} \leq 4 \sqrt{s} \\\|\delta\|_{2} \leq 1}} \sum_{k=1}^{d_{2}} V_{k} \delta_{k}\right]+4 \nu \sqrt{\frac{s}{d_{2}}},
$$

Applying Theorem 5.1(ii) from Gordon et al. 13] with $\left(q_{0}, q_{1}\right)=(1,2)$, $n=d_{2}$ and $t=4 \sqrt{s}$ then yields

$$
\mathbb{E}[\widetilde{Z}(s)] \leq c \frac{\nu}{\sqrt{d_{1} d_{2}}} \sqrt{s} \sqrt{2+\log \left(\frac{2 d_{2}}{16 s}\right)}+4 \nu \sqrt{\frac{s}{d_{2}}},
$$

which combined with the concentration bound (76) yields the refined claim.

Case 2: $\quad$ Alternatively, we may assume that $\left\|\widehat{\Delta}^{\Gamma}\right\|_{2,1}>\sqrt{s}\left\|\widehat{\Delta}^{\Gamma}\right\|_{\mathrm{F}}$. In this case, we need to verify that the choice (75) $\mu_{d}$ satisfies $\mu_{d}\left\|\widehat{\Delta}^{\Gamma}\right\|_{2,1} \geq 2\left|\left\langle\left\langle W, \widehat{\Delta}^{\Gamma}\right\rangle\right\rangle\right|$ with high probability. We have the upper bound

$$
\mid\left\langle\left\langle W, \widehat{\Delta}^{\Gamma}\right\rangle\left|\leq \sup _{\substack{\|\Delta\|_{2,1} \leq\left\|\widehat{\Delta}^{\Gamma}\right\|_{2,1} \\\|\Delta\|_{\mathrm{F}} \leq\left\|\widehat{\Delta}^{\Gamma}\right\|_{\mathrm{F}}}}\right|\langle\langle W, \Delta\rangle\rangle\right|=\left\|\widehat{\Delta}^{\mathrm{T}}\right\|_{\mathrm{F}} \widetilde{Z}\left(\frac{\left\|\widehat{\Delta}^{\Gamma}\right\|_{2,1}}{\left\|\widehat{\Delta}^{\Gamma}\right\|_{\mathrm{F}}}\right),
$$

where for any radius $t>0$, we define the random variable

$$
\widetilde{Z}(t):=\sup _{\substack{\|\Delta\|_{2,1} \leq t \\\|\Delta\|_{\mathrm{F}} \leq 1}}|\langle\langle W, \Delta\rangle\rangle| \text {. }
$$


Following through the same argument as in Case 2 of Appendix 10.1 yields that for any fixed $t>0$, we have

$$
\widetilde{Z}(t) \leq c \frac{\nu}{\sqrt{d_{1} d_{2}}} t \sqrt{2+\log \left(\frac{2 d_{2}}{t^{2}}\right)}+4 \nu \frac{t}{\sqrt{d_{2}}}+2 \nu t \sqrt{\frac{\log \left(\frac{d_{2}}{s}\right)}{d_{1} d_{2}}}
$$

with high probability. As before, this can be extended to a uniform bound over $t$ by a peeling argument, and we conclude that

$$
\begin{aligned}
\left|\left\langle W, \widehat{\Delta}^{\Gamma}\right\rangle\right| & \leq\left\|\widehat{\Delta}^{\Gamma}\right\|_{\mathrm{F}} \widetilde{Z}\left(\frac{\left\|\widehat{\Delta}^{\Gamma}\right\|_{2,1}}{\left\|\widehat{\Delta}^{\Gamma}\right\|_{\mathrm{F}}}\right) \\
& \leq c\left\|\widehat{\Delta}^{\Gamma}\right\|_{2,1}\left\{\frac{\nu}{\sqrt{d_{1} d_{2}}} \sqrt{2+\log \left(\frac{2 d_{2}}{\left\|\widehat{\Delta}^{\Gamma}\right\|_{2,1}^{2} /\left\|\widehat{\Delta}^{\Gamma}\right\|_{\mathrm{F}}^{2}}\right)}+4 \nu \frac{1}{\sqrt{d_{2}}}+2 \nu \sqrt{\frac{\log \left(\frac{d_{2}}{s}\right)}{d_{1} d_{2}}}\right\}
\end{aligned}
$$

with high probability. Since $\frac{1}{\left\|\widehat{\Delta}^{\Gamma}\right\|_{2,1}^{2} /\left\|\widehat{\Delta}^{\Gamma}\right\|_{\mathrm{F}}^{2}} \leq \frac{1}{s}$ by assumption, the claim follows.

11. Proof of Lemma 4. Since $\widehat{\Theta}$ and $\Theta^{\star}$ are optimal and feasible (respectively) for the convex program (41), we have

$$
\frac{1}{2}\|Y-\widehat{\Theta}-\widehat{\Gamma}\|_{\mathrm{F}}^{2}+\lambda_{d}\|\widehat{\Theta}\|_{\mathrm{N}} \leq \frac{1}{2}\left\|Y-\Theta^{\star}-\widehat{\Gamma}\right\|_{\mathrm{F}}^{2}+\lambda_{d}\left\|\Theta^{\star}\right\|_{\mathrm{N}} .
$$

Recalling that $Y=\Theta^{\star}+\Gamma^{\star}+W$ and re-writing in terms of the error matrices $\widehat{\Delta}^{\Gamma}=\widehat{\Gamma}-\Gamma^{\star}$ and $\widehat{\Delta}^{\Theta}=\widehat{\Theta}-\Theta^{\star}$, we find that

$$
\frac{1}{2}\left\|\widehat{\Delta}^{\Theta}+\widehat{\Delta}^{\Gamma}-W\right\|_{\mathrm{F}}^{2}+\lambda_{d}\left\|\Theta^{\star}+\widehat{\Delta}^{\Theta}\right\|_{\mathrm{N}} \leq \frac{1}{2}\left\|\widehat{\Delta}^{\Gamma}-W\right\|_{\mathrm{F}}^{2}+\lambda_{d}\left\|\Theta^{\star}\right\|_{\mathrm{N}} .
$$

Expanding the Frobenius norm and reorganizing terms yields

$$
\frac{1}{2}\left\|\widehat{\Delta}^{\Theta}\right\|_{\mathrm{F}}^{2} \leq\left|\left\langle\left\langle\widehat{\Delta}^{\Theta}, \widehat{\Delta}^{\Gamma}+W\right\rangle\right\rangle\right|+\lambda_{d}\left\{\left\|\Theta^{\star}\right\|_{\mathrm{N}}-\lambda_{d}\left\|\Theta^{\star}+\widehat{\Delta}^{\Theta}\right\|_{\mathrm{N}}\right\} .
$$

From Lemma 1 in the paper [22], there exists a decomposition $\widehat{\Delta}^{\Theta}=\widehat{\Delta}_{A}^{\Theta}+$ $\widehat{\Delta}_{B}^{\Theta}$ such that the rank of $\widehat{\Delta}_{A}^{\Theta}$ upper-bounded by $2 r$ and

$$
\left\|\Theta^{\star}\right\|_{\mathrm{N}}-\left\|\Theta^{\star}+\widehat{\Delta}_{A}^{\Theta}+\widehat{\Delta}_{B}^{\Theta}\right\|_{\mathrm{N}} \leq 2 \sum_{j=r+1}^{d} \sigma_{j}\left(\Theta^{\star}\right)+\left\|\widehat{\Delta}_{A}^{\Theta}\right\|_{\mathrm{N}}-\left\|\widehat{\Delta}_{B}^{\Theta}\right\|_{\mathrm{N}},
$$


which implies that

$$
\begin{aligned}
\frac{1}{2}\left\|\widehat{\Delta}^{\Theta}\right\|_{\mathrm{F}}^{2} & \leq\left|\left\langle\left\langle\widehat{\Delta}^{\Theta}, \widehat{\Delta}^{\Gamma}+W\right\rangle\right\rangle\right|+\lambda_{d}\left\{\left\|\widehat{\Delta}_{A}^{\Theta}\right\|_{\mathrm{N}}-\left\|\widehat{\Delta}_{B}^{\Theta}\right\|_{\mathrm{N}}\right\}+2 \lambda_{d} \sum_{j=r+1}^{d} \sigma_{j}\left(\Theta^{\star}\right) \\
& \stackrel{(i)}{\leq}\left|\left\langle\widehat{\Delta}^{\Theta}, \widehat{\Delta}^{\Gamma}\right\rangle\right|+\left|\left\langle\left\langle\widehat{\Delta}^{\Theta}, W\right\rangle\right\rangle\right|+\lambda_{d}\left\|\widehat{\Delta}_{A}^{\Theta}\right\|_{\mathrm{N}}-\lambda_{d}\left\|\widehat{\Delta}_{B}^{\Theta}\right\|_{\mathrm{N}}+2 \lambda_{d} \sum_{j=r+1}^{d} \sigma_{j}\left(\Theta^{\star}\right) \\
& \stackrel{(i i)}{\leq}\left\|\widehat{\Delta}^{\Theta}\right\|_{\mathrm{F}} \delta+\left\|\widehat{\Delta}^{\Theta}\right\|_{\mathrm{N}}\|W\|_{\mathrm{op}}+\lambda_{d}\left\|\widehat{\Delta}_{A}^{\Theta}\right\|_{\mathrm{N}}-\lambda_{d}\left\|\widehat{\Delta}_{B}^{\Theta}\right\|_{\mathrm{N}}+2 \lambda_{d} \sum_{j=r+1}^{d} \sigma_{j}\left(\Theta^{\star}\right) \\
& \stackrel{(i i i)}{\leq}\left\|\widehat{\Delta}^{\Theta}\right\|_{\mathrm{F}} \delta+\|W\|_{\mathrm{op}}\left\{\left\|\widehat{\Delta}_{A}^{\Theta}\right\|_{\mathrm{N}}+\left\|\widehat{\Delta}_{A}^{\Theta}\right\|_{\mathrm{N}}\right\}+\lambda_{d}\left\|\widehat{\Delta}_{A}^{\Theta}\right\|_{\mathrm{N}}-\lambda_{d}\left\|\widehat{\Delta}_{B}^{\Theta}\right\|_{\mathrm{N}}+2 \lambda_{d} \sum_{j=r+1}^{d} \sigma_{j}\left(\Theta^{\star}\right) \\
& =\left\|\widehat{\Delta}^{\Theta}\right\|_{\mathrm{F}} \delta+\left\|\widehat{\Delta}_{A}^{\Theta}\right\|_{\mathrm{N}}\left\{\|W\|_{\mathrm{op}}+\lambda_{d}\right\}+\left\|\widehat{\Delta}_{B}^{\Theta}\right\|_{\mathrm{N}}\left\{\|W\|_{\mathrm{op}}-\lambda_{d}\right\}+2 \lambda_{d} \sum_{j=r+1}^{d} \sigma_{j}\left(\Theta^{\star}\right),
\end{aligned}
$$

where step (i) follows by triangle inequality; step (ii) by the Cauchy-Schwarz and Hölder inequality, and our assumed bound $\left\|\widehat{\Delta}^{\Gamma}\right\|_{\mathrm{F}} \leq \delta$; and step (iii) follows by substituting $\widehat{\Delta}^{\Theta}=\widehat{\Delta}_{A}^{\Theta}+\widehat{\Delta}_{B}^{\Theta}$ and applying triangle inequality.

Since we have chosen $\lambda_{d} \geq\|W\|_{\mathrm{op}}$, we conclude that

$$
\begin{aligned}
\frac{1}{2}\left\|\widehat{\Delta}^{\Theta}\right\|_{\mathrm{F}}^{2} & \leq\left\|\widehat{\Delta}^{\Theta}\right\|_{\mathrm{F}} \delta+2 \lambda_{d}\left\|\widehat{\Delta}_{A}^{\Theta}\right\|_{\mathrm{N}}+2 \lambda_{d} \sum_{j=r+1}^{d} \sigma_{j}\left(\Theta^{\star}\right) \\
& \leq\left\|\widehat{\Delta}^{\Theta}\right\|_{\mathrm{F}} \delta+2 \lambda_{d} \sqrt{2 r}\left\|\widehat{\Delta}^{\Theta}\right\|_{\mathrm{F}}+2 \lambda_{d} \sum_{j=r+1}^{d} \sigma_{j}\left(\Theta^{\star}\right)
\end{aligned}
$$

where the second inequality follows since $\left\|\widehat{\Delta}_{A}^{\Theta}\right\|_{\mathrm{N}} \leq \sqrt{2 r}\left\|\widehat{\Delta}_{A}^{\Theta}\right\|_{\mathrm{F}} \leq \sqrt{2 r}\left\|\widehat{\Delta}^{\Theta}\right\|_{\mathrm{F}}$. We have thus obtained a quadratic inequality in $\left\|\widehat{\Delta}^{\Theta}\right\|_{F}$, and applying the quadratic formula yields the claim. 


\section{REFERENCES}

[1] A. Agarwal, S. Negahban, and M. J. Wainwright. Supplemental file to Noisy Matrix Decomposition via Convex Relaxation: Optimal Rates in High Dimensions. Technical report, University of California, Berkeley, 2012.

[2] T. W. Anderson. An Introduction to Multivariate Statistical Analysis. Wiley Series in Probability and Mathematical Statistics. Wiley, New York, 2003.

[3] R. K. Ando and T. Zhang. A framework for learning predictive structures from multiple tasks and unlabeled data. J. Mach. Learn. Res., 6:1817-1853, December 2005.

[4] P. J. Bickel and E. Levina. Covariance regularization by thresholding. Annals of Statistics, 36(6):2577-2604, 2008.

[5] J. Blitzer, D. P. Foster, and Sham M. Kakade. Zero-shot domain adaptation: A multi-view approach. Technical report, Toyota Technological Institute at Chicago, 2009.

[6] J. Blitzer, R. Mcdonald, and F. Pereira. Domain adaptation with structural correspondence learning. In EMNLP Conference, 2006.

[7] S. Boyd and L. Vandenberghe. Convex optimization. Cambridge University Press, Cambridge, UK, 2004.

[8] E. J. Candes, X. Li, Y. Ma, and J. Wright. Robust Principal Component Analysis? Technical report, Stanford, 2009. available at arXiv:0912.3599.

[9] V. Chandrasekaran, P. A. Parillo, and A. S. Willsky. Latent variable graphical model selection via convex optimization. Technical report, Massachusetts Institute of Technology, 2010.

[10] V. Chandrasekaran, S. Sanghavi, P. A. Parrilo, and A. S. Willsky. Rank-sparsity incoherence for matrix decomposition. Technical report, MIT, June 2009. Available at arXiv:0906.2220v1.

[11] K. R. Davidson and S. J. Szarek. Local operator theory, random matrices, and Banach spaces. In Handbook of Banach Spaces, volume 1, pages 317-336. Elsevier, Amsterdam, NL, 2001.

[12] J. Fan, Y. Liao, and M. Mincheva. Large covariance estimation by thresholding principal orthogonal components. Technical report, Princeton University, 2012. arxiv1201.0175v1.

[13] Y. Gordon, A. E. Litvak, S. Mendelson, and A. Pajor. Gaussian averages of interpolated bodies and applications to approximate reconstruction. Journal of Approximation Theory, 149:59-73, 2007.

[14] R. Z. Has'minskii. A lower bound on the risks of nonparametric estimates of densities in the uniform metric. Theory Prob. Appl., 23:794-798, 1978.

[15] R. A. Horn and C. R. Johnson. Matrix Analysis. Cambridge University Press, Cambridge, 1985.

[16] D. Hsu, S. M. Kakade, and T. Zhang. Robust matrix decomposition with sparse corruptions. Technical report, Univ. Pennsylvania, November 2010.

[17] I. M. Johnstone. On the distribution of the largest eigenvalue in principal components analysis. Annals of Statistics, 29(2):295-327, April 2001.

[18] M. Ledoux. The Concentration of Measure Phenomenon. Mathematical Surveys and Monographs. American Mathematical Society, Providence, RI, 2001.

[19] J. Matousek. Lectures on discrete geometry. Springer-Verlag, New York, 2002.

imsart-aos ver. 2007/12/10 file: AOS_supp.tex date: June 5, 2012 
[20] M. McCoy and J. Tropp. Two Proposals for Robust PCA using Semidefinite Programming. Technical report, California Institute of Technology, 2010.

[21] S. Negahban, P. Ravikumar, M. J. Wainwright, and B. Yu. A unified framework for high-dimensional analysis of M-estimators with decomposable regularizers. In NIPS Conference, Vancouver, Canada, December 2009. Full length version arxiv:1010.2731v1.

[22] S. Negahban and M. J. Wainwright. Estimation of (near) low-rank matrices with noise and high-dimensional scaling. Annals of Statistics, 39(2):1069-1097, 2011.

[23] S. Negahban and M. J. Wainwright. Restricted strong convexity and (weighted) matrix completion: Optimal bounds with noise. Journal of Machine Learning Research, 2012. Originally posted as arxiv:1009.2118.

[24] Y. Nesterov. Gradient methods for minimizing composite objective function. Technical Report 76, Center for Operations Research and Econometrics (CORE), Catholic University of Louvain (UCL), 2007.

[25] G. Raskutti, M. J. Wainwright, and B. Yu. Minimax rates of estimation for high-dimensional linear regression over $\ell_{q}$-balls. IEEE Trans. Information Theory, 57(10):6976 - 6994, October 2011.

[26] G. Raskutti, M. J. Wainwright, and B. Yu. Minimax-optimal rates for sparse additive models over kernel classes via convex programming. Journal of Machine Learning Research, 12:389-427, March 2012. Originally posted as arxiv:1008.3654.

[27] B. Recht, M. Fazel, and P. Parrilo. Guaranteed minimum-rank solutions of linear matrix equations via nuclear norm minimization. SIAM Review, 52(3):471-501, 2010.

[28] R. T. Rockafellar. Convex Analysis. Princeton University Press, Princeton, 1970.

[29] A. Rohde and A. Tsybakov. Estimation of high-dimensional low-rank matrices. Annals of Statistics, 39(2):887-930, 2011.

[30] S. van de Geer. Empirical Processes in M-Estimation. Cambridge University Press, 2000.

[31] H. Xu, C. Caramanis, and S. Sanghavi. Robust PCA via Outlier Pursuit. Technical report, University of Texas, Austin, 2010. available at arXiv:1010.4237.

[32] Y. Yang and A. Barron. Information-theoretic determination of minimax rates of convergence. Annals of Statistics, 27(5):1564-1599, 1999.

[33] B. Yu. Assouad, Fano and Le Cam. In Festschrift for Lucien Le Cam, pages 423-435. Springer-Verlag, Berlin, 1997.

[34] M. Yuan, A. Ekici, Z. Lu, and R. Monteiro. Dimension reduction and coefficient estimation in multivariate linear regression. Journal Of The Royal Statistical Society Series B, 69(3):329-346, 2007.

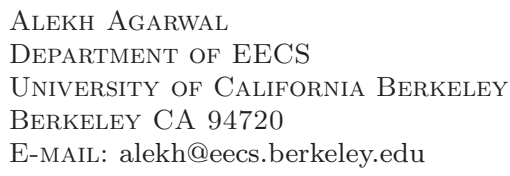

\author{
SAHAND N. NEGAHBAN \\ DEPARTMENT OF EECS \\ Massachusetts Institute of Technology \\ 32 Vassar Street \\ CAmbirdge MA 02139 \\ E-MAIL: sahandn@mit.edu
}

Martin J. WainWright

Department of EECS and Statistics

University of CALifornia Berkeley

BERKELEY CA 94720

E-MAIL: wainwrig@eecs.berkeley.edu 